\title{
ReMed: Jahresbericht 2017
}

ReMed, Leitungsausschuss

\section{ReMed - das Unterstützungsnetzwerk für Ärztinnen und Ärzte}

Ärztinnen und Ärzte arbeiten nach besten Kräften für die Erhaltung und Wiederherstellung der Gesundheit ihrer Patientinnen und Patienten.

Die eigene Gesundheit sollte jedoch ein genauso zentrales Anliegen sein. Denn bedingt durch ihre Arbeitssituation sind Ärztinnen und Ärzte besonderen Risikofaktoren ausgesetzt, welche zu physischen und psychischen Problemen führen können. ReMed ist ein Unterstützungsnetzwerk für Ärztinnen und Ärzte, das Wissen und Erfahrung über Gesundheitsförderung und Prävention verbreitet und Ärztinnen und Ärzte für die eigene Gesundheit sensibilisiert. Es bietet ein breites Angebot für Krisensituationen und hat zum Ziel, die Gesundheit und ärztliche Funktionalität zu erhalten sowie die Patientensicherheit und die hohe Qualität in der medizinischen Versorgung zu gewährleisten.

Kontext: ReMed ist eine ärzteeigene, von der FMH finanzierte, unabhängige Organisation und orientiert sich in seiner Vorgehensweise an den gesetzlichen Rahmenbedingungen sowie an der Standesordnung der FMH.

\section{ReMed - 141 Beratungsanfragen 2017}

Dass die Dienste von ReMed, dem Unterstützungsnetzwerk für Ärztinnen und Ärzte in Krisen, auch im vergangenen Jahr gefragt und notwendig waren, zeigen die 141 Beratungsanfragen, welche beim Netzwerk eingegangen sind. Gegenüber dem Vorjahr haben sich die Kontaktaufnahmen um ein Viertel vermehrt. Bei den Kontaktaufnahmen, welche von einem ärztlichen Mitglied des Leitungsausschusses beantwortet wurden, erfolgten 107 Anfragen aus der Deutschschweiz und 34 Fälle aus der Romandie. Die Kontaktsuchenden waren zu ca. $61 \%$ weiblich und ca. $39 \%$ männlich. Die Kontaktsuchenden verteilen sich etwa hälftig auf den ambulanten und stationären Bereich.

\section{ReMed - Rückmeldungen}

Ärztinnen und Ärzte, welche sich hilfesuchend an ReMed gewendet haben, nutzten die Möglichkeit, anonym die Qualität der Erstberatung zu bewerten. Die anonymisierten Rückmeldungen helfen, das Programm
ReMed weiterzuentwickeln und eine qualitative Verbesserung der Erstberatung zu ermöglichen.

\section{ReMed - externe Evaluation abgeschlossen}

Seit der Einführung von ReMed 2010 hat nach achtjährigem Bestehen eine externe Institution das Programm ReMed evaluiert, um so die Grundlage für Entscheidungen bezüglich der zukünftigen Ausgestaltung von ReMed zu schaffen. Im Rahmen der Evaluation äusserten sich über 90\% der befragten Ärztinnen und Ärzte, dass das Angebot von ReMed einem Bedürfnis der Ärzteschaft entspricht. Ebenfalls waren fast $90 \%$ der Kontaktsuchenden mit der Reaktionszeit von ReMed zufrieden. Aufgezeigt wurde auch, dass ReMed seine Ziele bezüglich Krisenintervision und Prävention erreicht.

\section{ReMed - Programmadäquanz}

Angebote, Bedürfnisse und Organisation von ReMed sind aufeinander abgestimmt. ReMed ist bestrebt, die Angebote stetig zu optimieren und zu erweitern. 2017 konnte das ReMed-Netzwerk verstärkt und vergrössert werden. Erstberatende, Netzwerkmitglieder, weiterbetreuende Fachpersonen haben sich mehrmals im Jahr getroffen und die Möglichkeit für Fallvorstellungen und Erfahrungsaustausch genutzt. Die grosse Teilnahme führt dazu, dass 2018 zusätzliche Intervisionstreffen und Erfahrungsaustausche zwischen den Erstberatenden angeboten werden.

\section{ReMed - Coaching-Gruppen}

Das 2016 gestartete Pilotprojekt «Coaching-Gruppen» konnte 2017 erfolgreich weitergeführt werden. In Bern und Zürich fanden Gesprächsrunden unter fachlicher, kompetenter Anleitung, die in einem kollegialen Verhältnis Unterstützung für den herausfordernden Berufsalltag bieten, statt. Das Angebot richtet sich an Ärztinnen und Ärzte, die mit belastenden, schwierigen und herausfordernden Themen konfrontiert worden sind, mit dem Ziel, arbeitsbezogene Konflikte und Schwierigkeiten zu thematisieren und entsprechende Lösungen zu finden. Auch 2018 werden Gruppen in Bern, Zürich und St. Gallen angeboten. 


\section{ReMed - präsent sein}

Dank zahlreichen Auftritten an Kongressen im In- und Ausland konnte ReMed den Bekanntheitsgrad weiter ausbauen. Das Unterstützungsnetzwerk repräsentierte mit Workshops und Darbietungen sein Angebot und leistete damit weiter einen grossen Beitrag zur Enttabuisierung der beruflichen ärztlichen Belastungen. Auch in den Medien war ReMed 2017 präsent und konnte entsprechende Artikel veröffentlichen. Ebenfalls konnte mit den erfolgreichen Erfahrungsberichten (Testimonials) in der Schweizerischen Ärztezeitung aufgezeigt werden, wie ReMed Betroffenen geholfen hat. Diese Artikel finden jeweils grossen Anklang bei der Leserschaft und erfahren positive Rückmeldungen.

\section{ReMed - Homepage in neuem Kleid}

Im vergangenen Jahr wurde die Homepage von ReMed angepasst und aufgefrischt. Besonders stolz ist man auf den erarbeiteten Clip, welcher den Homepage-Besucher auf der Startseite begrüsst und Gründe für eine Kontaktaufnahme zu ReMed zeigt.

Besuchen Sie die Homepage www.swiss-remed.ch und finden Sie interessante Erfahrungsberichte und Dokumentationen sowie alle wertvollen Informationen über das Unterstützungsnetzwerk.

Das nationale Programm ReMed mit dem Leitungsausschuss und den Netzwerkmitgliedern wird auch im Jahr 2018 für Ärztinnen und Ärzte in Krisensituationen da sein. Die betroffenen Ärztinnen und Ärzte können sich über die 24-Stunden-Hotline (0800 0 73633) oder per help@swiss-remed.ch melden.

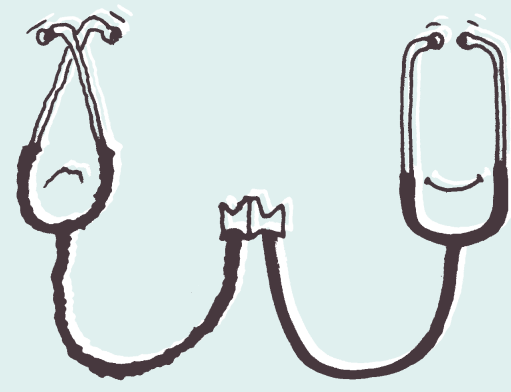

Plötzlich selbst betroffen. Krisen treffen auch Ärztinnen und Ärzte.

Lassen Sie sich helfen. Kontaktieren Sie ReMed.

24-Stunden-Hotline 0800073633

help@swiss-remed.ch

08000 ReMed www.swiss-remed.ch 